\title{
Evidence for intermittency in a granular medium: experiments and simulations.
}

\author{
Malte Schmick and Mario Markus \\ Max-Planck-Institut für molekulare Physiologie, Postfach 500 247, D-44202 Dortmund, Germany
}

\begin{abstract}
We present the first experimental demonstration of intermittency in a granular medium. The medium consists of magnets embedded within spheres. These spheres are placed in a horizontal Petri dish where they roll by virtue of an alternating, homogenous magnetic field. Due to collisions with the wall, clustering leads to self-organization into ring pieces circulating along the wall. The intermittent behaviour consists of an aperiodical alternation of this circular motion with a gaslike state extended over the entire dish. Molecular dynamic simulations agree with observations.
\end{abstract}

Keywords: granular media, intermittency, self-organization

\section{Introduction}

Intermittent behaviour, in the sense that a system aperiodically abandons given dynamic modes and return to them, has been shown to follow from various mechanisms in a large number of (nongranular) systems: one-dimensional maps [Fra92], coupled maps [DY96], single particles in particular potentials [LG95, SO96], electrical circuits [KPKA95], a mechanical system involving springs [WM99], a model applicable to stellar activity [CT97], optical systems [SK96], and discharge plasmas [SMW95]. In granular media, this phenomenon is known only as a result of computer simulations [SGM00, SGM02]. In the present contribution we shall present measurements of such a phenomenon, which to our knowledge are the fir rst evidence of intermittency in a granular medium.

In granular media, the dissipation of energy due to collisions is balanced by an external source. In most systems investigated so far, a subset of the particles receives energy from a wall, as unique external source, and transfers this energy to the other particles via collisions. Such systems have been called "elitary" systems [PLMV99]; examples are: rotating drums [MSMO95, ZLL+94, SFT99, SAM99], vertical vibrating plates [BSS ${ }^{+} 98$, UMS96] and swirling dishes [KGM99, $\mathrm{SKM}^{+}$00]. In contrast, energy is transfered from outside to each particle indiscriminately in the system examined in the present work, so that we are dealing with a so-called "democratic" system [PLMV99]. Simulations in one [WM96, SBCM98] and in two dimensions [Wil96] of "democratic" media have yielded spatially irregular coexistence of hot, gaslike clusters with cool, liquidlike regions. Experimentally, a two-dimensional "democratic" medium has been implemented by a three-meter-high device consisting of a vertical wind tunnel bringing about a homogeneous cushion of air on a horizontal porous slab over which the granular medium, consisting of 1365-8050 (c) 2003 Discrete Mathematics and Theoretical Computer Science (DMTCS), Nancy, France 
flat disks, is placed [IAL $\left.{ }^{+} 95\right]$; the failure of equipartition of energy in a binary mixture was demonstrated with that device. We implemented a "democratic" system by embedding magnetic dipoles in spheres and placing these spheres in a homogenous, alternating magnetic fi eld. This approach was also proposed recently in [CLH02].

\section{Experiments}

Alternating magnetic fi elds were generated using a coil with a vertically aligned axis. This coil had 20 cylindrical, concentric layers of copper wire. (Number of circular windings per layer: 40; wire diameter: $0.27 \mathrm{~cm}$, including $0.2 \mathrm{~mm}$ of isolating material; thus, height of the coil: $40 \times 0.27 \mathrm{~cm}=10.8 \mathrm{~cm}$.) The inner radius of the coil was $6.75 \mathrm{~cm}$ and the outer one was $12.25 \mathrm{~cm}$. Currents $\tilde{I}=I_{0} \sin (2 \pi f t), I_{0}<10 A$, $1 \mathrm{~Hz}<f<30 \mathrm{~Hz}$ were applied. We measured magnetic inductions $B_{0}=4.7 \cdot 10^{-3} I_{0}\left(B_{0}\right.$ in Tesla, $I_{0}$ in Ampere). $B_{0}$ varied less than $2 \%$ in horizontal direction within a distance of $5 \mathrm{~cm}$ from the center of the coil; in the vertical direction, the fi eld varied less than $0.4 \%$ within the diameter $\phi$, of a sphere.

A (round) Petri dish (diameter: $\phi_{d}$ ) was placed horizontally at the center of the coil. The bottom of the dish was covered with a billiard felt. The granular material consisted of spheres, each of which was constructed by gluing together two hemispheres with holes drilled so as to hold a well-centered magnet. The spheres were painted black for better visualization. The magnets were obtained by punching round holes (with a ticket punch; diameter: $1 \mathrm{~mm}$ or $2 \mathrm{~mm}$ ) into magnetic rubber plates (Flexo 180 from Schallenkammer, Rimpar, Germany; thickness: $1.5 \mathrm{~mm}$; density: $3.7 \mathrm{~g} / \mathrm{cm}^{3}$; remanence: $B_{R}=265 \mathrm{mT}$ ). We used two types of spheres: i) wood spheres (mass, including the magnet: $110 \mathrm{mg}$; diameter: $\phi_{s}=6 \mathrm{~mm}$; length of magnet: $L_{M}=2 \times 1.5 \mathrm{~mm}$; magnet diameter: $\phi_{M}=2 \mathrm{~mm}$ ); and ii) PVC spheres (mass, including the magnet: 49mg; diameter: $\phi_{s}=4 \mathrm{~mm}$; length of magnet: $L_{M}=1.5 \mathrm{~mm}$; magnet diameter: $\phi_{M}=1 \mathrm{~mm}$ ). A sketch of the dish with the spheres, the magnets and the fi eld is given in Fig. 1.

The movement of the spheres in the dish was monitored by a CCD camera connected to video equipment and a PC. The particle velocities were determined by evaluating two succesive images with a time difference of $1 / 25 \mathrm{~s}$. Each velocity distribution was determined out of 500 velocity measurements.

\section{Simulations}

Calculations were performed using three-dimensional molecular dynamics [Hai92]. Each sphere is described by its position (two components), velocity (two components), orientation of the dipole (three components) and angular velocity (three components). For the calculations of the dipole-dipole and dipole-fi eld interactions, we used (in analogy to the treatment of electrical dipoles) the computational approach in which it is assumed that there exist poles (points at the centers of the flat faces of the cylindrical magnets) having strength $P=B_{R} A$ ( $B_{R}$ : remanence of the magnetic material; A: area of the flat faces of the magnets). Another approximation was the assumption of a homogeneous mass distribution leading to a scalar moment of inertia.

The force acting between two poles is given by $\vec{F}= \pm\left(P^{2} / x^{2}\right) \hat{x}$, where $\hat{x}$ is the unit vector along the 
line containing the poles, $x$ is the distance, and the sign indicates attraction or repulsion. The corresponding torque is $\vec{M}=\vec{F} \times \vec{L}$, where $\vec{L}=\left(L_{M} / 2\right) \hat{L}, \hat{L}$ being the unit vector along the dipole. The fi eld-dipole interaction causes a torque $\vec{M}=2 P \vec{L} \times \vec{H}$, where $\vec{H}=\left(B_{0} / \mu_{0}\right) \sin (2 \pi f t) \hat{e}_{z}, \hat{e}_{z}$ being the unit vector in vertical direction and $\mu_{0}$ the permittivity constant. Since we considered the magnetic interaction of every sphere with every other one, our present computational limitations forced us to restrict the number of spheres to $N=6$.

For interactions that do not involve magnetic fi elds, we set the forces as in our previous simulations [SGM00, SGM02, $\left.\mathrm{SKM}^{+} 00\right]$. However, we adjusted some parameters by roughly fi tting simulations to experiments, accounting for the specifi $\mathrm{c}$ features in the present work. By virtue of this adjustment, we set the coeffi cient of sliding friction to $f_{s}=0.9$ for the sphere-felt interaction at the bottom of the dish and $f_{s}=0.45$ for all other interactions, the damping constant to $\gamma_{n}=50 \mathrm{~kg} \mathrm{~m}^{-1 / 2} \mathrm{~s}^{-1}$, and the viscous flow coeffi cient to $\gamma=10 \mathrm{~kg} \mathrm{~s}^{-1}$. Furthermore, we introduced a translatory rolling friction (as in [KGM99]; $\left.\mu_{r}=3 \cdot 10^{-5} \mathrm{~m}\right)$ and a rotational rolling friction $-\mu_{R} \omega_{z} \hat{e}_{z}\left(\omega_{z}\right.$ : vertical component of the sphere's angular velocity), setting $\mu_{R}=10^{-9} \mathrm{~kg} \mathrm{~m} / \mathrm{s}$.

\section{Results}

Both in experiments and in simulations, we found two dynamic modes: i) a gaslike attractor (exemplifi ed in Fig. 2); and ii) intermittency (Fig. 3 and 4) between a gaslike transient and a circular motion, in which all spheres revolve as ring pieces along the border of the dish. A necessary condition for this latter behaviour is that the number of spheres is lower than the densest single row along the border. This condition is, of course, not fulfi lled in Fig. 2. The ring pieces at the border in Fig. 2 are extremely short-lived, since they become disrupted within times $\lesssim 0.2$ s by collisions with spheres farther inside. In Figs. 4 and 5 , we compare experimental observations of intermittency with simulations. The nearly constant value $d \approx \phi_{s} / 2$ corresponds to the circular motion. The two circulating directions (see Figs. 3 and 4 ) are unpredictable and equally probable.

Fig. 6a shows measured velocity distribution functions (for $N=6$ ), as compared to simulations in Fig. 6b. The continuous curve shows the probability distribution of the magnitude of the velocities of the spheres. The dashed curve was obtained by considering only the movement of spheres when their center was farther away from the border than $2 / 3$ of the sphere's diameter.

\section{Discussion and conclusions}

Comparison of the dashed curves with the continuous curves in Fig. 6 shows that the velocity distribution is strongly inhomogeneous, favouring low velocities in the vicinity of the border of the dish. In other words, emergent "elitary" conditions due to the wall are signifi cant in our system, although we have set "democratic" control conditions by leaving out energy supply from any wall. In our system, the "elitary" behaviour is due to collisions of the spheres with the wall, turning the latter into an energy sink that considerably affects the system's homogeneity. Differences between simulations and experiments in Fig. 6, especially in the scaling of the abscissa, may be due to errors in our estimations of the model parameters, 
as well as due to our approximations that the magnets are one-dimensional dipoles and that the moment of inertia is a scalar.

As a consequence of the density enhancement in the region close to the border, we found that clustering can take place in that region. However, this clustering becomes organized into circulating ring pieces, contrary to the highly disordered clustering reported in Refs. [SGM02, WM96, Wi196]. The sketch in Fig. 7 explains the self-organization of a ring piece in our system. For the explanation, two spheres are suffi cient. The sphere in the back (left part of the picture) points into a gap between the wall and the sphere moving in front of it, and thus gets trapped. Eventually, a ring piece gets disrupted for two reasons: i) The trapping is metastable (see Fig. 7); and ii) a chaotic motion (arising from the coupling of oscillators; see e.g.[Moo87]) is superposed to the ordered circular motion. The continuous ellipses drawn within the spheres in Fig. 7 are typical trajectories of one of the poles of the magnet. The dashed ellipse is a typical deviation of such a pole's trajectory, leading to motion of the sphere away from the dish's border. Thereafter, globally disrupting scenarios can occur; an example is shown in Fig. 3a through 3g: a sphere (in that fi gure the last sphere of the circulating assembly) shortcuts across the dish, collides with the ring and thus disrupts it into a gaslike transient. Note that the chaotic motion superposed (with lower amplitude) to the ordered circular confi guration causes time dependent gaps within the ring, leading to breaking and merging of ring pieces (see Fig. 3a through 3d).

Intermittency here is characterized by gaslike and circulating confi gurations that have no long-term stability; for this reason and because no other confi gurations are attained, two-state on-off intermittency takes place (similarly to that described in Ref. [LG95] for a simple particle in a potential). To our knowledge, this is the fi rst demonstration of intermittency in a granular medium. In previous works, intermittency was predicted for disks moving in a vertical double-window that is periodically tilted around the lower vertex [SGM00, SGM02]; however, there has been no success in implementing that system experimentally because the double-window must be so narrow (to confi ne the disks into two dimensions) that friction impedes intermittency. In contrast, the confi guration in the present work allows two-dimensional confi nement without prohibitively large friction.

\section{Acknowledgements}

We thank the Deutsche Forschungsgemeinschaft (Grant Ma 629/6) for fi nancial support.

\section{References}

[BSS ${ }^{+}$98] C. Bizon, M.D. Shattuk, J.B. Swift, W.D. McCormick, and H.L. Swinney. Patterns in 3d vertically oscillated granular layers: simulations and experiments. Phys. Rev. Lett., 89:5760, 1998 .

[CLH02] R. Cafi ero, S. Luding, and H.J. Herrmann. Rotationally driven gas of inelastic rough spheres. Europhys. Lett., 60:854-860, 2002.

[CT97] E. Covas and R. Tavakol. Crisis-induced intermittency in truncated mean fi eld dynamos. Phys. Rev. E, 55:6641-6645, 1997. 
[DY96] M.Z. Ding and W.M. Yang. Observation of intermingled basins in coupled oscillators exhibiting synchronized chaos. Phys. Rev. E, 54:2489-2494, 1996.

[Fra92] M. Franaszek. Analyzing chaotic behaviour via multitransient chaos. Phys. Rev. A, 46:63406343, 1992.

[Hai92] J.M. Haile. Molecular Dynamics Simulations. Wiley, New York, 1992.

[IAL ${ }^{+95] ~ I . ~ I p p o l i t o, ~ C . ~ A n n i c, ~ J . ~ L e m a i t r e, ~ L . ~ O g e r, ~ a n d ~ D . ~ B i d e a u . ~ G r a n u l a r ~ t e m p e r a t u r e: ~ e x p e r i-~}$ mental anlysis. Phys. Rev. E, 52:2072-2075, 1995.

[KGM99] K. Kötter, E. Goles, and M. Markus. Shell structures with 'magic numbers' of spheres in a swirled dish. Phys. Rev. E, 60:7182-7185, 1999.

[KPKA95] I.M. Kyprianidis, M.L. Petrani, J.A. Kalomiros, and A.N. Anagnostopoulos. Crisis-induced intermittency in a third-order electrical circuit. Phys. Rev. E, 52:2268-2273, 1995.

[LG95] Y.-C. Lai and C. Grebogi. Intermingled basins and two-state on-off intermittency. Phys. Rev. E, 52:3313-3316, 1995.

[Moo87] F.C. Moon. Chaotic Vibrations. J. Wiley \& Sons, New York, 1987.

[MSMO95] G. Metcalfe, T. Shinbrot, J.J. McCarthy, and J.M. Ottino. Avalanche mixing of granular solids. Nature, 374:39-41, 1995.

[PLMV99] A. Puglisi, V. Loreto, V. Marini Bettolo Marconi, and A. Vulpiani. Kinetic approach to granular gases. Phys. Rev. E, 59:5582-5595, 1999.

[SAM99] T. Shinbrot, A. Alexander, and F.J. Muzzio. Spontaneous chaotic granular mixing. Nature, 397:675-678, 1999.

[SBCM98] M.R. Swift, M. Boamfa, S.J. Cornell, and A. Marita. Scale invariant correlations in a driven dissipative gas. Phys. Rev. Lett., 80:4410-4413, 1998.

[SFT99] A.Q. Shen, E. Fried, and S.T. Thoroddsen. Is seggregation-by-particle type a generic mechanism underlying fi nger formation at fronts of flowing granular media? Particulate Science and Technology, 17:141-147, 1999.

[SGM00] M. Schmick, E. Goles, and M. Markus. Intermingled basins due to fi nite accuracy. Phys. Rev. E, 62:397-401, 2000.

[SGM02] M. Schmick, E. Goles, and M. Markus. On-off intermittency and intermingledlike basins in a granular medium. Phys. Rev. E, 066214:1-4, 2002.

[SK96] M. Sauer and F. Kaiser. Crisis-induced intermittency in a spatially extended nonlinear optical system. Int. J. Bifurcation Chaos Appl. Sci. Eng., 6:1481-1494, 1996.

$\left[\mathrm{SKM}^{+}\right.$00] M.A. Scherer, K. Kötter, M. Markus, E. Goles, and I Rehberg. Swirling granular solidlike clusters. Phys. Rev. E, 61:4069-4077, 2000. 
[SMW95] H.Y. Sun, L.X. Ma, and L. Wang. Multistability as an indicator of chaos in a discharge plasma. Phys. Rev. E, 51:3475-3479, 1995.

[SO96] J.C. Sommerer and E. Ott. Intermingled basins of attraction: uncomputability in a simple physical system. Phys. Lett. A, 214:243-251, 1996.

[UMS96] P.B. Umbanhowar, F. Melo, and H.L. Swinney. Localized excitations in a vertically vibrated granular layer. Nature, 382:793-796, 1996.

[Wi196] D.R.M. Williams. Driven granular media and dissipative gases: correlations and liquid-gas phase transitions. Physica A, 233:718-729, 1996.

[WM96] D.R.M. Williams and F.C. MacKintosh. Driven granular media in one dimension: correlations and equation of state. Phys. Rev. E, 54:R9-R12, 1996.

[WM99] M. Woltering and M. Markus. Riddled basins of coupled elastic arches. Phys. Lett. A, 260:453-461, 1999.

$\left[\mathrm{ZLL}^{+}\right.$94] O. Zik, D. Levine, S.G. Lipson, S. Shtrikman, and J. Stavans. Rotationally induced seggregation of granular materials. Phys. Rev. Lett., 73:644-647, 1994. 


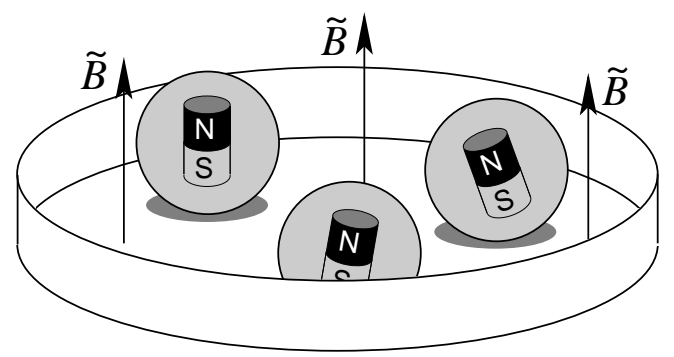

Fig. 1: Sketch of the spheres and the dish in which they move by virtue of their embedded magnets and an alternating magnetic field $\tilde{B}$. (For clarity, the size of the spheres is shown here exagerately larger than the dish). $\tilde{B}$ is generated by a coil (not drawn) that sorrounds the setup coaxially to the dish and is dimensioned so as to guarantee that $\tilde{B}$ is sufficiently homogeneous.

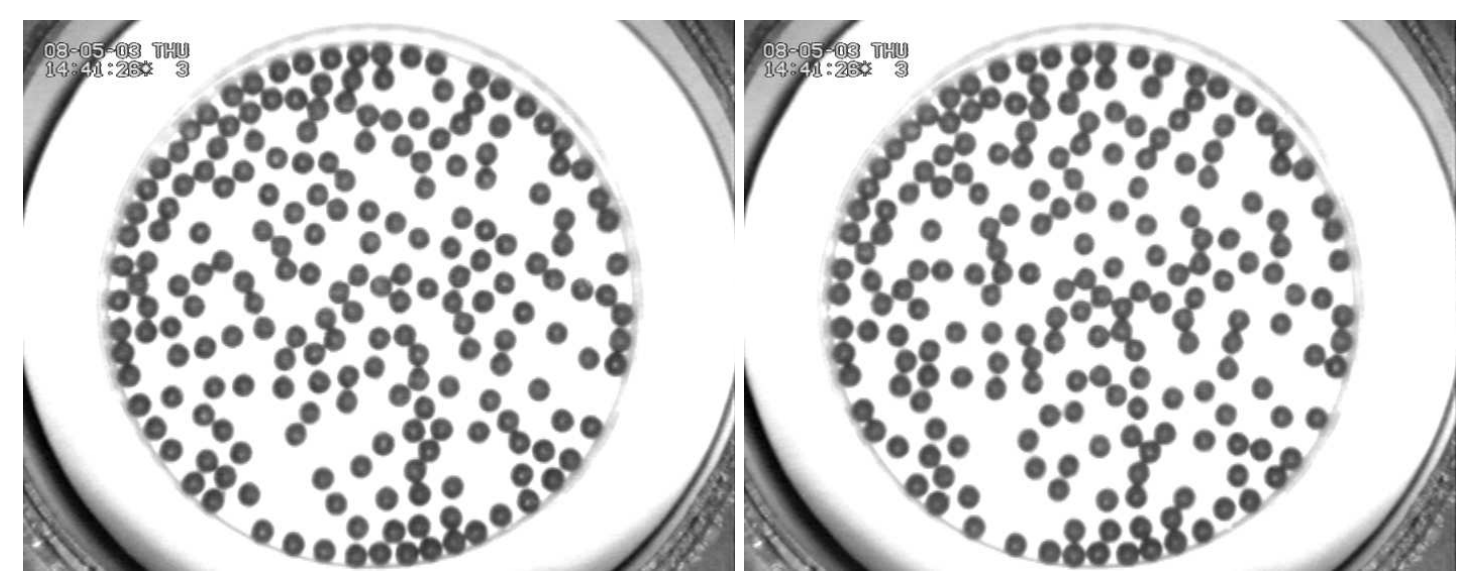

Fig. 2: a) Experimental observations of $250 \mathrm{PVC}$ spheres in the gaslike mode: $\phi_{s}=4 \mathrm{~mm}, \phi_{d}=9 \mathrm{~cm}, B_{0}=12.5 \mathrm{mT}$, $f=15 \mathrm{~Hz}$. We show consecutive frames (1/25 s apart), $15 \mathrm{~s}$ after starting the experiment. 
a)

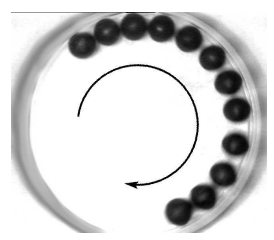

e)

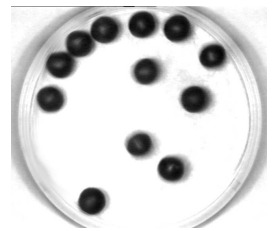

b)

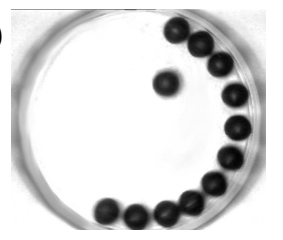

f)

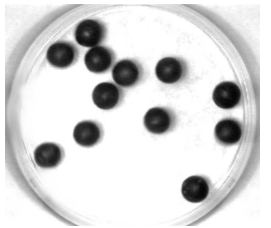

c)

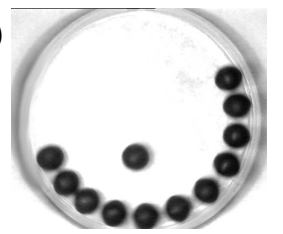

g)

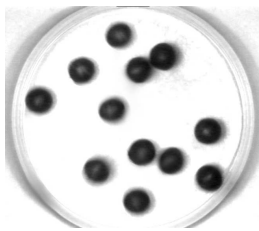

d)

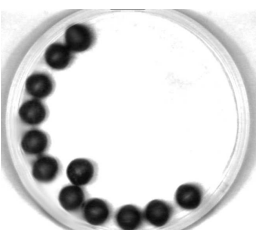

h)

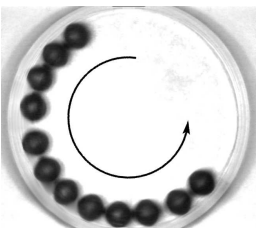

Fig. 3: Experimental observations of 12 wood spheres in the intermittent mode: $\phi_{s}=6 \mathrm{~mm}, \phi_{d}=5 \mathrm{~cm}, B_{0}=9.3 \mathrm{mT}$, $f=10 \mathrm{~Hz}$. Times: a) $36.3 \mathrm{~s}$, b) $36.5 \mathrm{~s}$, c) $36.7 \mathrm{~s}$, d) $36.9 \mathrm{~s}$, e) $37.4 \mathrm{~s}$, f) $37.9 \mathrm{~s}$, g) $38.9 \mathrm{~s}$, h) $50 \mathrm{~s}$. The first seven pictures illustrate a transition from circular to gaslike motion. Arrows: circulating directions.

a)
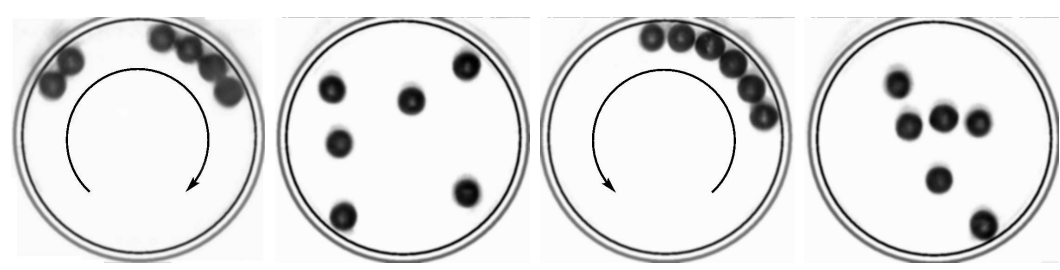

b)
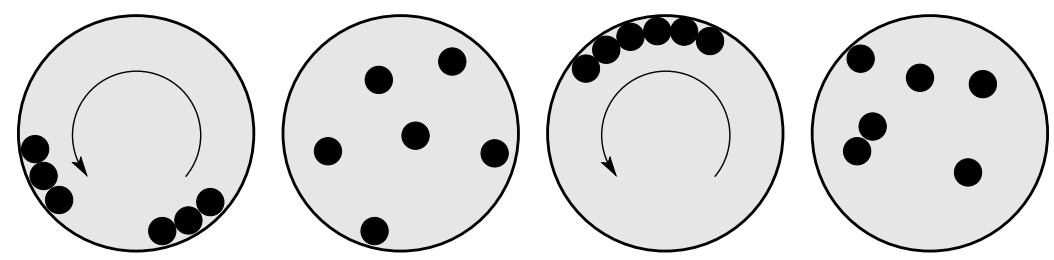

Fig. 4: a) Experimental observations of 6 wood spheres in the intermittent mode; $\phi_{s}=6 \mathrm{~mm}, \phi_{d}=5 \mathrm{~cm}, B_{0}=9.3 \mathrm{mT}$, $f=10 \mathrm{~Hz}$. b) Corresponding simulations; arrows: circulating direction. Times (left to right): $55 \mathrm{~s}, 75 \mathrm{~s}, 120 \mathrm{~s}, 145 \mathrm{~s}$.

a)

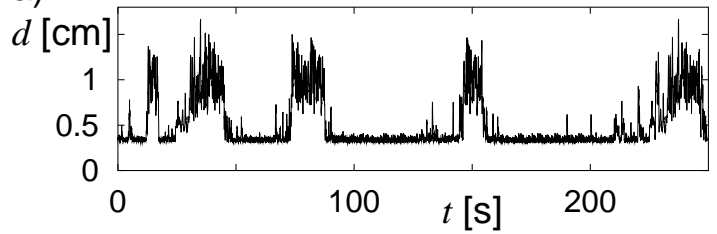

b)

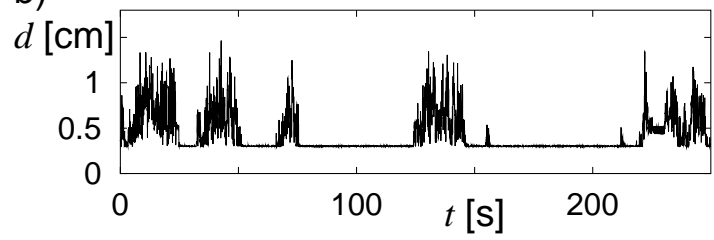

Fig. 5: Shortest distance $d$ between the center of the spheres and the border of the dish, averaged over all spheres, as a function of time $t$. a) Experiments corresponding to Fig. 4a. b) Simulations corresponding to Fig.4b. 

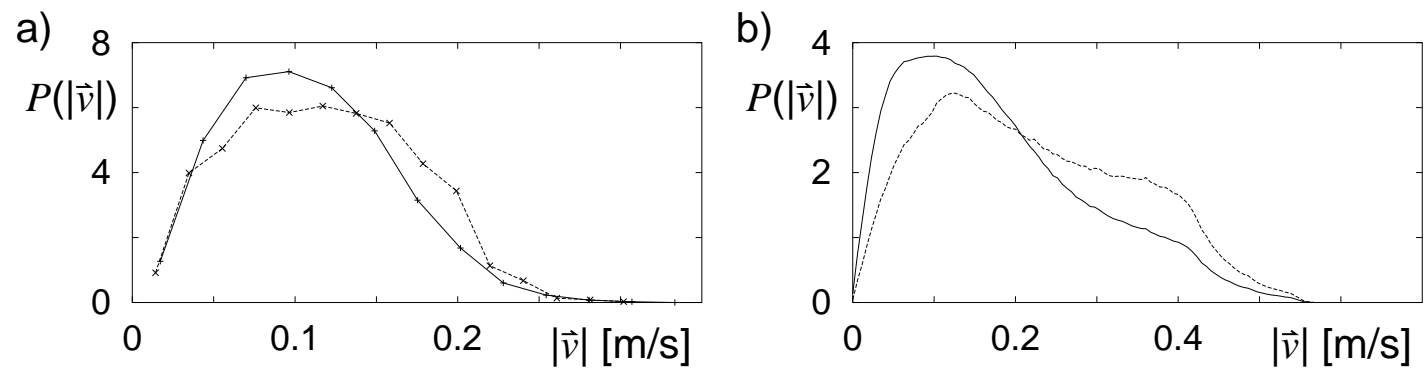

Fig. 6: Experimental distribution of the magnitudes of the velocities. Continuous curve: evaluation over the whole dish. Dashed curve: distribution with the constraint that the distances between the sphere's centers and the border of the dish are larger than $2 / 3 \phi_{s} . \phi_{s}=6 \mathrm{~mm}, \phi_{d}=5 \mathrm{~cm}, B_{0}=9.3, f=10 \mathrm{~Hz}$. b) Corresponding simulations.

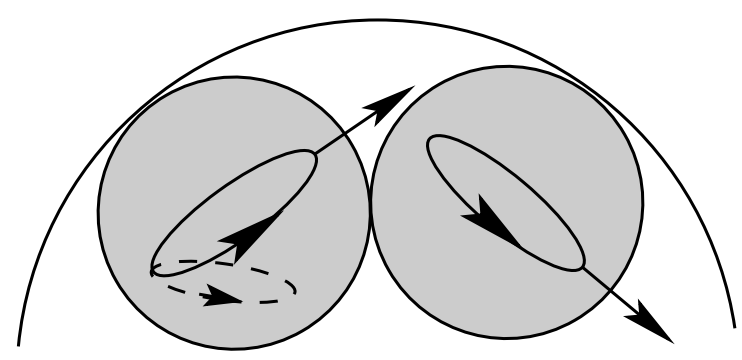

Fig. 7: Scheme illustrating the movement of spheres belonging to a ring piece. Straight arrows: direction of movement of single spheres. Continuous ellipses: trajectories of one of the magnetic poles. Dashed ellipse: trajectory of the magnetic pole shortly before the sphere at the left ceases to circulate along the border of the dish. 
